\title{
Avaliação de Músicas Compostas para Indução de Relaxamento e de seus Efeitos Psicológicos
}

\author{
Marília Nunes-Silva \\ Universidade Federal de Minas Gerais, BH, Brasil. \\ Gerlaine Teixeira Rosa \\ Ana Carolina Duarte Valadares \\ Fundação de Saúde Integral Humanística, BH, Brasil. \\ Liliane Cristina Moreira Lopes \\ Fundação de Saúde Integral Humanística, BH, Brasil. $\quad$ Fundação de Saúde Integral Humanística, BH, Brasil. \\ Célia Auxiliadora dos Santos Marra \\ Fundação de Saúde Integral Humanística, BH, Brasil.
}

Resumo: O presente estudo teve por objetivo avaliar as características das músicas compostas por dois diferentes compositores para a fase preparatória da Abordagem Direta do Inconsciente/Terapia de Integração Pessoa (ADI/TIP) e seus efeitos psicológicos, buscando verificar se elas contribuem para a indução de relaxamento. Para tanto, foi realizada inicialmente a análise teórica das músicas com o intuito de verificar se apresentavam características estruturais consideradas relaxantes, evidenciadas pelos estudos experimentais revisados. Posteriormente, as músicas foram avaliadas considerando os efeitos subjetivos eliciados pela sua audição. Participaram do estudo 72 voluntários, divididos em quatro condições: 1) Compositor A; 2) Compositor B; 3) Coletânea (músicas utilizadas em estudos prévios para promover o relaxamento); e 4) Sem audição musical. Os participantes responderam a um protocolo de avaliação da percepção subjetiva de sentimentos e emoções antes e depois de serem expostos à condição experimental. Os dados do protocolo foram comparados com os dados obtidos na análise teórica das músicas. Apesar de as músicas do compositor A apresentarem mais características estruturais relaxantes do que as do compositor B, todas as músicas analisadas foram consideradas como relaxantes e tiveram efeito para redução da pontuação da categoria de "Raiva/Medo" do protocolo utilizado. Isso indica que as músicas utilizadas na fase preparatória do método ADI/TIP possuem características relaxantes e contribuem para diminuição de sensações correspondentes a uma valência negativa e excitabilidade alta, favorecendo à promoção do estado de relaxamento necessário à eficácia deste método terapêutico.

Palavras-chave: Música, Relaxamento, Efeitos psicológicos, ADI / TIP. 


\title{
Evaluation of Music Composed for Inducing Relaxation and its Psychological Effects
}

\begin{abstract}
The present study aimed to evaluate the characteristics of songs composed by two different composers for the preparatory stage of the ADI/TIP and its psychological effects, seeking to ensure that these songs contribute to relaxation induction. To this end, we initially held a theoretical analysis of these songs in order to verify if they presented structural characteristics considered as relaxing by some experimental studies reviewed. The subjective effects elicited by the audition of these songs were subsequently evaluated. For this purpose, 72 volunteers participated in this study, distributed into four different conditions: 1) Composer A; 2) Composer B; 3) Compilation album (songs used in previous studies to promote relaxation); and 4) No music listening condition. Participants answered a protocol for assessing their subjective perception of feelings and emotions before and after being exposed to the experimental conditions. We compared the protocol data with data obtained from the theoretical analysis of the songs. Despite songs composed by Composer A present more relaxing structural characteristics than Composer B's songs, all of these songs could be considered as relaxing and had effect to reduce the score of the "Anger/Fear" category of the protocol utilized. This indicates that songs used in the preparatory phase of the ADI method/TIP have relaxing characteristics and contributed to decrease the sensations corresponding to a negative valence and high excitability, favoring the promotion of relaxation necessary for the effectiveness of this therapeutic method.
\end{abstract}

Keywords: Music, Relaxation, Psychological effects, ADI / TIP.

\section{Evaluación de Canciones Compuestas para la Inducción a la Relajación y sus Efectos Psicológicos}

Resumen: El presente estudio tuvo por objetivo evaluar las características de canciones compuestas por dos diferentes compositores para la fase preparatoria de la ADI/TPI y sus efectos psicológicos, buscando verificar si ellas contribuyen para la inducción a la relajación. Para tanto, se realizó inicialmente el análisis teórico de las canciones con la intención de verificar si presentaban características estructurales consideradas relajantes, evidenciadas por los estudios experimentales revisados. Posteriormente, las canciones fueron evaluadas considerando los efectos subjetivos causados por su audición. Participaron del estudio 72 voluntarios, divididos según cuatro condiciones: 1) Compositor A; 2) Compositor B; 3) Colección (canciones utilizadas en estudios previos para promover la relajación); y 4) Sin audición musical. Los participantes contestaron a un protocolo de evaluación de la percepción subjetiva de sentimientos y emociones antes y después de la exposición a la condición experimental. Los datos del protocolo fueron comparados con los obtenidos en el análisis teórico de las canciones. A pesar de que las músicas del compositor A presentaron más características estructurales relajantes que las del compositor B, todas las canciones analizadas fueron consideradas relajantes y resultaron en la reducción de la puntuación de la categoría de "Rabia/Miedo" del protocolo utilizado. Esto indica que las canciones utilizadas en la fase preparatoria del método ADI/TIP poseen características relajantes y contribuyen a la disminución de sensaciones relacionadas a una valencia negativa y excitabilidad alta, lo que favorece la promoción del estado de relajación necesario para la eficacia de este método terapéutico.

Palabras clave: Música, Relajación, Efectos psicológicos, ADI / TIP 


\section{Introdução}

No âmbito da Psicologia e saúde, as técnicas de relaxamento se configuram como importantes estratégias utilizadas durante o processo terapêutico, tendo sido consolidadas como procedimentos válidos de intervenção psicológica ao longo do século XX (Vera, \& Vila, 2002). Segundo Souza, Forgione e Alves (2000), as técnicas de relaxamento podem ser consideradas como um conjunto de procedimentos úteis tanto no âmbito da Psicologia clínica quanto no âmbito da Psicologia aplicada a hospitais. Para estas autoras, o processo de relaxamento contribui para que o paciente modifique suas relações com seu corpo, com outras pessoas e com suas próprias emoções, sendo assim uma oportunidade de reorganizar e reintegrar o que é percebido como desestruturado. A música tem se inserido como uma das técnicas de relaxamento, uma vez que seus efeitos psicofisiológicos podem contribuir para a redução do estresse e dos níveis de cortisol (Nuki, Yoshiuchi, \& Nomura, 1999). Algumas abordagens dentro da Medicina, da Enfermagem, da Psicologia, da Musicoterapia e da área de saúde em geral se utilizam da música enquanto técnica de relaxamento (Good, 1995; Gruzzelier, 2002; Peterson, \& Silva, 2006; Wang, Kulkarni, Dolev, \& Kain, 2002). Em um estudo de revisão bibliográfica realizado no período de 1994 a 2004, Ferreira, Remedi e Lima (2006) verificaram que a música tem sido amplamente utilizada como recurso terapêutico e que é percebida como forma peculiar de indução de relaxamento.

Estudos indicam que a música utilizada para a promoção de relaxamento tem consequências positivas em processos terapêuticos de indivíduos em diferentes condições. Há evidências de resultados positivos em relação ao uso de intervenções musicoterapêuticas na redução de ansiedade em pacientes terminais (Horne-Thompson, \& Grocke, 2008) e na redução do nível de estresse de mulheres profissionais de saúde (Taets, Borba-Pinheiro, Figueiredo, \& Dantas, 2013). Evidências preliminares também sugerem que a Musicoterapia pode ser clinicamente útil para promover a sensação de relaxamento e aumento da atividade do sistema nervoso parassimpático em pessoas que sobreviveram ao câncer (Chuang, Han, Li, \& Young, 2010) e na promoção de relaxamento em pacientes transplantados (Madson, \& Silverman, 2010). Intervenções utilizando música também têm contribuído para promoção de relaxamento e redução de ansiedade em pacientes com queimaduras (Najafi Ghezeljeh,
Mohades Ardebili, Rafii, \& Haghan, 2016; Tan, Yowler, Super, \& Fratianne, 2010) e em idosos, com efeitos positivos sobre a depressão e a ansiedade (Klainin-Yobas, Oo, Suzanne Yew, \& Lau, 2015).

Dentro dessecontexto, observa-sequea utilização da música pode auxiliar na promoção do relaxamento e consequente facilitação de processos terapêuticos. É com essa perspectiva que o método da Abordagem Direta do Inconsciente (ADI) se utiliza da música em sua fase preparatória. De acordo com Moraes (2009), a metodologia de tratamento oferecido pela ADI consiste em acessar o inconsciente de maneira direta e consciente, com o intuito de investigar o seu conteúdo para realizar a recuperação da saúde integral dos pacientes. Moraes (2007) explica que a ansiedade pela cura, as somatizações e os desgastes psicoemocionais que sempre acompanham as pessoas que decidem fazer terapia precisam ser minimizados para uma melhor efetividade da terapia. No contexto terapêutico da ADI é necessário, portanto, que o paciente em terapia esteja treinado para desligar-se dos ruídos externos e concentrar-se em seu interior, a fim de que possa perceber com menos interferência, ou seja, sem racionalização, os conteúdos de seu inconsciente. Dessa forma, com base nessas necessidades, prevê-se dentro deste processo uma fase preparatória, na qual o cliente é levado para um ambiente propício onde é submetido à audição de uma música acompanhada de sugestões de imagens positivas, visando à indução de relaxamento.

De acordo com Vera e Vila (2002), o relaxamento se constitui em um processo psicofisiológico de caráter interativo, que deve levar em conta três aspectos: fisiológico, subjetivo e comportamental. O componente fisiológico se refere a um padrão reduzido de ativação somática e autonômica. O componente subjetivo é identificado por relatos verbais de tranquilidade e sossego. Já o componente comportamental é relacionado a um estado de tranquilidade motora. Dentro deste contexto, a música se insere como um dos instrumentos utilizados para a indução de relaxamento, uma vez que promove alterações desejadas nos três componentes supracitados.

Estudos indicam que a música provoca efeitos fisiológicos de redução do estresse, seguida da indução de relaxamento. Nuki et al. (1999) investigaram os efeitos da musicoterapia sobre mudanças dos hormônios relacionados ao estresse. Participaram do estudo 10 voluntários saudáveis, cinco 
mulheres e cinco homens, os quais foram submetidos à audição de cinco diferentes tipos de música. Inicialmente foi feito um estudo piloto no qual os participantes deveriam marcar em uma escala de avaliação de sete pontos composta de 18 pares de adjetivos opostos qual adjetivo se relacionava à música ouvida e em que intensidade. Foi realizada uma análise fatorial que revelou três categorias maiores: relaxante, vívida e apreciativa (relacionada a adjetivos positivos como interessante, célebre, natural). Foram observadas diferenças de gênero e de idade para a categorização dos diferentes tipos de música. Posteriormente, os participantes, após 15 minutos de descanso, ouviram durante 15 minutos as músicas selecionadas. Amostras de sangue, para medir os níveis de adrenalina, noradrenalina, dopamina, corticotropina e cortisol, foram retidas antes e depois da audição das músicas e após 15 minutos de descanso posterior à sessão de música. Nuki et al. (1999) observaram diminuições significativas dos níveis de noradrenalina, corticotropina e cortisol durante a audição de música indicando que a música pode ter efeitos sobre mudanças endocrinológicas que conduzem a um estado de relaxamento.

Kreutz, Bongard, Rohrmann, Hodapper e Grebe (2004), por sua vez, investigaram os efeitos da música coral (Réquiem de Mozart) sobre a secreção de imunoglobulina A e cortisol, e sobre o estado emocional dos membros de um coro amador misto em duas condições diferentes: cantando e ouvindo. O estado emocional foi mensurado a partir do Positive and Negative Affect Schedule-PANAS e os níveis de cortisol e imunoglobulina A foram medidos a partir de amostras de saliva dos participantes após as duas sessões (canto e audição, com intervalo de uma semana entre estas sessões) e depois de 60 minutos do final de cada sessão. Os autores encontraram que o canto coral favorece a um aumento significativo da imunoglobulina A e do afeto positivo, ao mesmo tempo em que o afeto negativo é reduzido. Já a audição da peça coral conduziu a um aumento do afeto negativo e diminuição dos níveis de cortisol. Mais estudos são necessários para investigar as diferenças encontradas entre as duas condições e para controlar os efeitos da música específica utilizada.

Em um artigo de revisão, Krout (2007) avaliou o papel da audição de música para facilitar o relaxamento e promover a saúde e o bem-estar. Seus estudos revelam que os estímulos cerebrais eliciados pela música podem ter efeito positivo sobre as funções neurais, a atividade hormonal e o sistema límbico. O autor evidencia os estudos referentes às respostas emocionais e neurofisiológicas suscitadas pela audição de música. De acordo com Krout (2007), mesmo que a pessoa não tome consciência de que está ouvindo uma música, esta já pode estar estimulando substâncias bioquímicas ou inibindo reações emocionais negativas no cérebro, através do sistema límbico. O sistema límbico está localizado no lobo temporal do cérebro, próximo ao córtex auditivo, onde a música e o som são transformados, e inclui ainda uma série de estruturas como o tálamo, a amígdala, o hipotálamo e outros. As respostas do tálamo aos ritmos musicais facilitam o relaxamento, afetando positivamente a frequência cardíaca, a respiração e a pressão arterial. Já a amígdala é responsável pelas respostas comportamentais aos estímulos percebidos pelo indivíduo, o que é de grande importância à captação do estímulo musical. O hipocampo, por sua vez, desenvolve um papel importante na memória, o que pode ser de grande utilidade quando a música ouvida for associada com os sentimentos anteriores de estado de relaxamento.

Segundo Krout (2007), a música pode afetar também o humor global e estimular a produção de endorfinas naturais que agem no organismo controlando os níveis de excitação, estimulando a sensação de prazer, reduzindo o estresse, facilitando o relaxamento e, consequentemente, provocando um funcionamento mais eficaz do sistema imunológico. Krout (2007), por fim, ressalta que o corpo humano tem uma pré-disposição natural para responder internamente, por meio de processos fisiológicos, aos sons e ritmos vindos do ambiente externo, o que permite a interação do sistema nervoso simpático com sistema nervoso parassimpático e facilita uma resposta de relaxamento indicando, portanto, que a música tem um papel significante na promoção do relaxamento.

Outro importante estudo dos efeitos relaxantes da música foi realizado por Khalfa, Dalla-Bella, Roy, Peretz e Lupien (2003), no qual foi constatado que algumas melodias particularmente calmas e harmoniosas diminuem no sangue a quantidade de cortisol, que é o hormônio liberado nesses momentos e que prepara o organismo para a fuga ou o combate. A partir da monitoração de mudanças do nível de cortisol salivar, observou-se que o nível de cortisol após o evento estressante parou de aumentar naqueles estu- 
dantes que ouviram música, enquanto que, nos estudantes que somente ficaram em silêncio, o nível de cortisol continuou a aumentar durante 30 minutos. Pôde-se observar também que o resultado foi independente da preferência musical dos estudantes, o que pode indicar que características das músicas utilizadas agiriam sobre estruturas cerebrais homogêneas entre diferentes indivíduos. Khalfa et al. (2003) sugerem, a partir disso, que músicas que comportam disparidades de ritmo e dissonâncias podem ser estressantes. Segundo a hipótese de Khalfa et al. (2003), passado o evento de estresse, uma música caracterizada pela lentidão, regularidade de tempo e harmonia ativa o córtex auditivo juntamente com outras estruturas implicadas no trato das emoções, reduzindo a atividade do complexo amigdalóide, parando de liberar o cortisol e inibindo toda a cadeia de reações. A música pode proporcionar assim, uma sensação de bem-estar e alívio, cortando a cadeia de reações que ocorre devido a um evento estressante. A audição de músicas durante e após cirurgias tem se mostrado eficaz para a redução dos níveis de cortisol (Chanda, \& Levitin, 2013; Koelsch et al., 2011), porém há controvérsias em relação à audição musical antes de um evento estressante (Koelsch et al., 2011; Thoma et al., 2013), indicando que os resultados da utilização da música para este fim possam depender também da cronologia dos acontecimentos (antes $v s$. durante $v s$. depois da condição estressante).

Além das características das músicas relaxantes observadas no estudo de Khalfa et al. (2003), Krout (2007) indica que, apesar de haver influência da preferência musical na percepção subjetiva de relaxamento, há alguns elementos musicais considerados quase universais para a indução de relaxamento. Esses elementos auxiliariam na escolha de músicas para a indução de relaxamento e são, a saber: tempo estável e lento, volume baixo, dinâmica leve com pouca variação, timbre suave, combinação harmoniosa de instrumentos, melodias com legatos (com notas ligadas, sem interrupção), harmonia e progressões de acordes mais simples e ausência de ritmo acentuado. Krout (2007) considera também que é frequentemente funcional iniciar o processo com elementos mais ativos e ritmo mais rápido, acompanhando o nível de atividade e estresse da pessoa e aos poucos ir diminuindo em termos de tempo e complexidade, tornando-se mais relaxante.
De acordo com Bigand, Vieillard, Madurell, Marozeau e Dacquet (2005), as músicas que suscitam serenidade ou relaxamento são caracterizadas por um modo maior e andamento lento. $\mathrm{O}$ modo é definido como uma série de notas sucessivas organizadas segundo um padrão de determinados intervalos e $o$ andamento como o grau de velocidade que se imprime à execução de um trecho musical. De acordo com os autores, considerando a abordagem categórica de emoções musicais, pode-se agrupar as emoções suscitadas por estímulos musicais em quatro tipos diferentes: a) tristeza - caracterizada por trechos musicais em modo menor e andamento lento; b) raiva/medo - caracterizados por um modo menor e andamento rápido; c) serenidade/relaxamento - caracterizados por um modo maior e andamento lento e d) alegria - provocada por músicas em modo maior e andamento rápido. Porém, em seu estudo, Bigand et al. (2005) relatam que os participantes distinguiam os trechos musicais em um número maior de categorias, o que favorece a uma abordagem multidimensional na qual as emoções suscitadas pela música se apresentam em contínuos que levam em conta a valência (positiva ou negativa) e a excitabilidade (alta ou baixa) emocional. Os autores evidenciam também que, apesar de associarmos as músicas a eventos pessoais, em geral, o reconhecimento das características emocionais da música é consistente entre indivíduos diferentes e no mesmo indivíduo, e independe do nível de educação musical.

Riccardi (2002), por sua vez, sintetiza as características potenciais das músicas relaxantes, que se mantêm de forma estável ou com pouca variação. Para a autora, a dinâmica deve apresentar-se de forma gradual e com acentos esporádicos ou pouco marcados. O tempo deve ser estável com mudanças graduais de tempo ou ritmo. $\mathrm{O}$ andamento é moderato (72-90 pulsações por minuto), seguindo o ritmo cardíaco normal. Considera também as estruturas métricas ternárias como mais relaxantes. A música deve ter forma e estrutura definidas, linhas melódicas, harmonias e cadências previsíveis, motivos e frases que se repetem e estabilidade harmônica e melódica com mudanças graduais. Os intervalos de terças, quintas, oitavas e sextas, as cadências perfeitas, os esquemas harmônicos I-V e a resolução cadencial devem favorecer o equilíbrio. Em relação ao timbre e textura musical, dá-se preferência a instrumentos agradáveis, sendo importante garantir a sensação de continuidade musical para a pessoa e que ela possa 
prever ou antecipar o estímulo sonoro em sequência. Os estudos acima evidenciam os aspectos psicofisiológicos da música para a promoção do relaxamento. Pode-se observar que as músicas consideradas relaxantes possuem algumas características em comum. A escolha da música para este fim deve ser, portanto, feita de maneira criteriosa, obedecendo a certos parâmetros como os que foram expostos acima.

A fase preparatória da ADI/TIP se constitui em uma etapa fundamental para o processo terapêutico, pois visa facilitar o acesso consciente ao inconsciente por meio da promoção do relaxamento e diminuição da ansiedade dos pacientes. Isto só se torna possível com a redução do estado de alerta e de abertura do paciente ao inusitado. Então, o objetivo da utilização da música na fase preparatória do Método ADI/TIP é promover o relaxamento através da quebra do estresse contínuo, diminuição da ansiedade, liberação de endorfinas e diminuição de cortisol a fim de se obter esse intento. Sendo assim, é importante investigar se os estímulos musicais utilizados na fase preparatória para a indução de relaxamento seriam adequados para o objetivo proposto. Sendo a música um dos elementos que podem potencializar o processo terapêutico, e dados os estudos que corroboram os seus efeitos psicofisiológicos para indução de relaxamento, torna-se necessário avaliar se as músicas utilizadas e compostas especificamente para a fase preparatória da ADI/TIP possuem as características adequadas para eliciar o efeito esperado e quais os benefícios psicológicos que elas causam em seus ouvintes. Sendo assim, o presente estudo teve como objetivo avaliar as características estruturais das duas músicas compostas para a fase preparatória da ADI/TIP a fim de verificar se elas se adéquam a essas necessidades terapêuticas.

\section{Método}

$\mathrm{O}$ estudo foi dividido em duas fases. A primeira fase constituiu-sena avaliação sistemática das músicas compostas para a fase preparatória da ADI/TIP tendo como parâmetro as características das músicas utilizadas para indução de relaxamento, evidenciadas pela revisão bibliográfica realizada. Já a segunda fase constituiu-se na avaliação dos efeitos subjetivos eliciados pela audição das músicas da fase preparatória da ADI/TIP e de músicas utilizadas em estudos prévios para promover o relaxamento.

\section{Participantes}

Participaram da primeira etapa dois profissionais graduados em música, responsáveis por fazer a análise qualitativa das músicas compostas para indução de relaxamento utilizadas na fase preparatória da ADI/TIP. Já a segunda etapa contou com a participação de 72 voluntários, com idades acima de 18 anos $(M=39,9, S D=12,6)$, sendo metade do gênero masculino e com escolaridade média de Ensino Superior incompleto $(\mathrm{M}=4,97, \mathrm{SD}=1,36$, considerando escala de 1 a 6 , sendo 1 correspondente a Ensino Fundamental incompleto e 6 para Ensino Superior completo). Os voluntários foram recrutados aleatoriamente na clínica da ADI/TIP através de um sorteio entre os pacientes agendados e que ainda não haviam iniciado o processo terapêutico, mantendo-se o critério estabelecido para a composição dos grupos de metade para cada gênero. Os participantes foram distribuídos aleatoriamente em quatro diferentes condições, sendo três experimentais, estabelecidas de acordo com o tipo de estímulo musical a ser escutado, e uma condição de controle, sem audição de músicas. As condições foram constituídas de 18 participantes cada e nomeadas da seguinte forma: 1) Compositor A, 2) Compositor B, 3) Coletânea e 4) Controle.

A Tabela 1 apresenta a caracterização da amostra apresentando a média e desvio padrão para idade e escolaridade, considerando as quatro diferentes condições. A partir do teste não paramétrico para amostras independentes Kruskal-Wallis, verificou-se que os quatro grupos não apresentaram diferenças estatisticamente significativas em relação à idade e escolaridade (valores $\chi 2$ e p da Tabela 1). Em relação ao gênero, para a composição dos grupos os participantes, foram selecionados de forma a manter a mesma proporção de homens e mulheres nove homens e nove mulheres para cada grupo.

Tabela 1

Dados para caracterização da amostra.

\begin{tabular}{lcc}
\hline Grupo & Idade & Escolaridade \\
\hline Compositor A & $38,5(10,0)$ & $5,11(1,64)$ \\
Compositor B & $40,33(11,99)$ & $4,72(1,32)$ \\
Coletânea & $45,11(15,72)$ & $4,83(1,42)$ \\
Controle & $35,89(11,19)$ & $5,22(1,06)$ \\
X2 & 3.841 & 2.964 \\
$\mathrm{P}$ & 0.279 & 0.397 \\
\hline
\end{tabular}




\section{Material}

1) Músicas compostas para indução de relaxamento: Foram utilizadas no presente estudo músicas especialmente compostas para a fase preparatória da ADI/TIP de dois diferentes compositores (aqui denominados Compositor A e Compositor B), sendo três músicas de cada compositor. Cada música tem duração de 50 minutos.

2) Coletânea de músicas relaxantes utilizadas em estudos prévios: foi utilizada uma coletânea de músicas cujos efeitos relaxantes foram evidenciados em estudos pregressos (Andrade, \& Ávila, 2007; Bigand et al., 2005; Chafin, Roy, Gerin, \& Christenfeld, 2004; Hatem, Lira, \& Mattos, 2006; Kain, Wang, Mayes, Krivutza, \& Teague, 2001; Primo, \& Amorim, 2008) e que correspondem às características das músicas relaxantes expostas na revisão bibliográfica do presente estudo. As músicas selecionadas foram: 1) Primavera do concerto "As quatro estações" deVivaldi (10min 20s); 2) Segundo movimento da Serenata Noturna (K239) de Mozart (4min 9s);3) Segundo movimento (Romance - Andante) da Serenata em Sol Maior (K 525) de Mozart (5min 44s); 4) Ária na corda sol (quarta corda) da Suíte orquestral $\mathrm{n}^{\circ} 3$ em Ré M (BWV1068) de Bach (4min 47s); e 5) Adágio do Concerto para Violino e orquestra em Ré maior (op. 77) de Brahms (9min 43s).

A duração total da coletânea é de 49 minutos e 48 segundos. Algumas músicas se repetem de modo que a coletânea se aproxime da condição das músicas utilizadas na fase preparatória da ADI/TIP, em relação ao tempo de duração. Para a ordenação das músicas, observou-se também a proposta de Krout (2007) de se iniciar o processo com elementos mais ativos e ritmo mais rápido, acompanhando o nível de atividade e estresse da pessoa e aos poucos ir diminuindo em termos de tempo e complexidade, tornando-se mais lento e relaxante. Portanto, a ordem de apresentação das músicas foi: 1) Primavera das Quatro Estações (Vivaldi);2) Segundo movimento (Romance - Andante) da Serenata (Mozart); 3) Segundo movimento da Serenata Noturna (Mozart); 4) Ária na quarta corda da Suíte orquestral no 3 (Bach); 5) Adágio do Concerto para Violino e orquestra (Brahms); 6) Ária na quarta corda da Suíte orquestral no 3 (Bach) e 7) Adágio do Concerto para Violino e orquestra (Brahms).

3) Escala Hospitalar de Ansiedade e Depressão (HADS): a HADS é um instrumento que avalia ansiedade e depressão, tendo se mostrado válido tanto para a população geral, quanto para pacientes hospi- talares, a partir de vários estudos (Botega, Bio, Zomignani, Garcia Junior, \& Pereira, 1995; Botega, Pondé, Medeiros, Lima, \& Guerreiro, 1998; Marcolino et al., 2007; Michopouloa et al., 2008; Snaith, 2003). A HADS é de fácil manuseio e de rápida execução e foi desenvolvida por Zigmond e Snaith em 1983 com a finalidade de fornecer um instrumento confiável e de fácil utilização para identificar e quantificar a depressão e a ansiedade (Michopoulos et al., 2008). É uma escala de autorrelato que possui 14 itens do tipo múltipla escolha em uma escala Likert de 4 pontos (de zero a três). Compõe-se de duas subescalas, sendo uma para ansiedade e outra para depressão, com sete itens cada. Solicita-se que a pessoa responda baseando-se em como se sentiu durante a última semana. Para a correção, a pontuação para cada resposta é dada na coluna da direita. A pontuação global em cada subescala é de 0 a 21 para cada uma (Marcolino et al., 2007; Michopoulos et al., 2008; Snaith, 2003;). A escala HADS encontra-se disponível na internet: http:// www.scielo.br/pdf/rba/v57n1/06.pdf.

4) Protocolo de avaliação de percepções subjetivas (PAPS): de acordo com Andrade e Gorestein (1998), em um estudo de revisão acerca do construto de ansiedade, o termo ansiedade abrange sensações e sentimentos diversos. Como não foi encontrado um instrumento de medida validado que considerasse $a$ ansiedade em seus diferentes aspectos ao mesmo tempo em que possibilitasse medir a ansiedade dos participantes antes e após a audição de músicas, bem como outras sensações e sentimentos percebidos por eles, optou-se por desenvolver o PAPS, especificamente para o presente estudo. O PAPS foi desenvolvido com intuito de apreender a percepção subjetiva dos sentimentos e sensações dos participantes em relação às músicas escutadas. $\mathrm{O}$ PAPS é dividido em duas partes, denominadas PAPS I e PAPS II, sendo o primeiro destinado a ser respondido na condição pré-teste e o segundo destinado a ser respondido na condição pós-teste. Ele se constitui em uma escala contendo uma seleção de 60 sentimentos ou sensações escolhidas e agrupadas aleatoriamente, com espaço entre parênteses para que os participantes marquem, dentre as opções, quais se aplicam ao seu momento presente. Os sentimentos e sensações foram classificados de acordo com a caracterização das quatro emoções básicas - alegria, tristeza, raiva/medo e serenidade - proposta por Bigand et al. (2005), com adaptações ao presente estudo. Objetivou-se definir os sentimentos que sugerem estado de relaxamento, sendo 
que, neste estudo, a categoria "serenidade" é utilizada como característica de relaxamento, diferenciando-os dos demais sentimentos. Bigand et al. (2005) apresentam as quatro emoções básicas utilizando de uma abordagem multidimensional de Russel (1980). Para melhor visualização, as emoções podem ser distribuídas em um gráfico onde o eixo vertical é representado pelo nível de excitação, o qual é baixo na parte inferior e alto na superior, e o eixo horizontal é representado pela valência emocional, sendo a valência positiva representada ao lado direito e a valência negativa à esquerda. A análise do grupo das quatro emoções básicas pode variar de acordo com a valência e excitação.

A Figura 1 demonstra que a alegria corresponderia a uma valência positiva e alta excitação; a tristeza seria representada por uma valência negativa e baixa excitação; Raiva/Medo corresponderiam a uma valência negativa e alta excitação; e a serenidade a uma valência positiva e baixa excitação.

Nesse contexto, as diversas emoções poderiam ser representadas dentro destes quatro blocos da Figura 1, com diferentes níveis de excitação e de valência. Portanto, a lista de sensações e sentimentos criada para o presente estudo foi classificada tendo-se por base estes quatro diferentes blocos. Sendo assim, uma sensação que corresponda a uma valência negativa e excitabilidade baixa, por exemplo, estaria classificada como tristeza. As sensações e sentimentos foram classificados conforme exposto na Tabela 2.

\section{Procedimentos}

Para a primeira etapa, os dois profissionais graduados em música foram convidados a fazerem a análise qualitativa das músicas compostas para indução de relaxamento. Para as análises, foi apresentada uma introdução sobre as características de músicas utilizadas para a promoção de relaxamento, evidenciadas em estudos empíricos prévios. A partir disto, os músicos avaliadores foram orientados a verificar se as músicas a serem analisadas apresentavam ou não estes elementos característicos de músicas relaxantes. As análises foram realizadas considerando-se os seguintes elementos musicais: ritmo, melodia, harmonia, timbre, composição dos instrumentos, andamento e dinâmica. Foram avaliadas seis músicas com duração de 50 minutos cada, sendo três músicas elaboradas pelo Compositor A e três pelo Compositor B. Para a segunda fase, porém, por possuírem características composicionais homogêneas, para cada compositor (A e B), foi utilizada apenas uma das três composições musicais.

Os participantes da segunda fase do estudo foram contatados inicialmente por telefone e informados a respeito dos procedimentos da pesquisa. Os indivíduos que manifestaram interesse em participar foram convidados a realizar os procedimentos em horários agendados. Os voluntários somente participaram da pesquisa após serem informados acerca dos procedimentos e que a experiência não lhes arrecadaria ônus prejudiciais ou outro de qualquer natureza. Na oportunidade, sua anuência foi confirmada

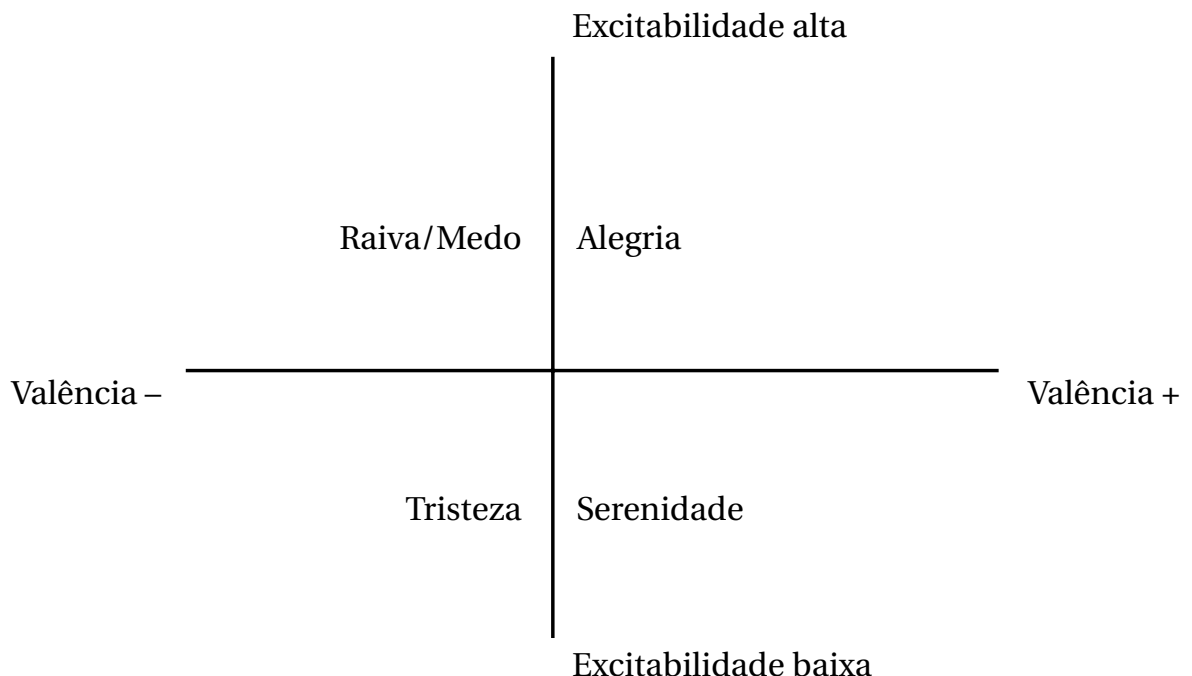

Figura 1

Categorização das quatro emoções básicas (adaptado de Bigand et al., 2005). 
Tabela 2

Categorização dos itens presentes no PAPS.

\begin{tabular}{|c|c|c|c|}
\hline Raiva/Medo & Alegria & Tristeza & Serenidade/Relaxamento \\
\hline 1- Agressividade & 1- Disposto & 1- Apatia & 1- Bem-estar \\
\hline 2- Medo & 2-Alegria & 2- Tédio & 2- Relaxado \\
\hline 3- Irritação & 3- Empolgado & 3- Tristeza & 3-Aliviado \\
\hline 4-Impaciente & 4- Euforia & 4- Angústia & 4- Corpo leve \\
\hline 5- Raiva & 5- Descontração & 5- Abafamento & 5- Serenidade \\
\hline 6- Estressado & 6-Vivacidade & 6- Pessimista & 6- Calma \\
\hline 7- Assustado & 7- Otimista & 7- Memórias negativas & 7- Tranquilidade \\
\hline 8- Nervoso & 8- Imagens positivas & 8- Vazio existencial & 8- Plenitude \\
\hline 9- Corpo pesado & 9- Memórias positivas & 9- Solidão & 9- Paz \\
\hline 10- Aflição & 10- Criatividade & 10- Imagens negativas & 10- Sentimento de religiosidade \\
\hline 11-Ansiedade & 11- Ânimo & 11-Abatimento & 11- Sentimento de elevação \\
\hline 12- Apreensivo & 12- Bem-humorado & 12-Amargura & 12- Confiança \\
\hline 13-Preocupado & 13- Satisfeito & 13- Sem energia & 13- Seguro \\
\hline 14- Tenso & 14- Êxtase & 14- Desamparado & 14- Confortável \\
\hline 15- Desconfortável & 15- Contentamento & 15- Desanimado & 15- Sossegado \\
\hline
\end{tabular}

PAPS: Protocolo de avaliação de percepções subjetivas.

mediante a assinatura do termo de consentimento livre e esclarecido, de acordo com o Comitê de Ética em Pesquisa (Protocolo do CEP: 326/2010). Os participantes foram distribuídos aleatoriamente nas quatro diferentes condições, sendo três experimentais, estabelecidas de acordo com o tipo de estímulo musical (Compositor A, Compositor B, Coletânea), e uma controle, sem audição de músicas. Em todas as condições eles responderam inicialmente ao HADS. Posteriormente, responderam ao PAPS antes e após serem expostos à condição a qual foram alocados. Cada condição teve duração de 50 minutos e foi realizada em salas disponibilizadas pela TIP Clínica e devidamente preparadas para garantir um ambiente propício ao estudo. Antes e após cada condição, os participantes foram instruídos a prestar atenção em suas sensações e sentimentos durante o tempo em que estivessem naquele ambiente. Foi mantido o mesmo volume para a audição de todas as músicas. Os participantes do grupo controle foram submetidos às mesmas avaliações e condições dos outros grupos, porém sem audição de música. Nos questionários realizados, foi atribuído a cada participante um número identificador para garantia do seu anonimato. A coleta de dados foi realizada pela pesquisadora responsável e por estagiários de Psicologia, previamente treinados.

\section{Resultados}

São apresentados os resultados para as duas fases do estudo. Foram realizadas análises qualitativas das músicas compostas para indução de relaxamento e análises quantitativas dos dados obtidos dos participantes.

\section{1) Análise qualitativa das músicas compostas para indução de relaxamento}

Foram analisadas seis músicas compostas para a fase preparatória da ADI/TIP, sendo três músicas de cada compositor (Compositor A e Compositor B), com duração de 50 minutos cada. A partir da análise realizada pelos músicos ${ }^{1}$, pode-se observar que as três músicas do compositor A possuem características composicionais semelhantes, com predominância de andamento lento e modo maior, característico de músicas relaxantes. Os ritmos são constantes, regulares e previsíveis. As melodias e harmonias são simples, havendo poucas dissonâncias e as modula-

${ }^{1}$ Para acesso à análise qualitativa detalhada, envie solicitação às autoras por e-mail. 
ções são sutis. Em geral, os timbres são suaves, a dinâmica não apresenta grandes mudanças e as variações ocorrem de maneira gradual.

Em relação às três músicas do compositor $\mathrm{B}$, a partir da análise qualitativa* também foi possível observar que as músicas possuem características composicionais similares, apesar das músicas serem menos homogêneas, quando comparadas entre si. Em geral, possuem andamento predominantemente lento e modo maior, mas modulam frequentemente para o modo menor. $\mathrm{O}$ ritmo apresenta-se regular, as melodias são simples, mas há várias modulações, apesar de a harmonia ser constituída de cadências simples. A dinâmica é estável em algumas partes e pode ser mais variável em outras, apresentando contrastes. Há muitas alterações de timbres, apesar dos timbres serem predominantemente suaves.

Comparativamente observou-se que as músicas do compositor A são mais constantes, sem grandes modificações e com timbres considerados mais relaxantes do que as músicas do compositor B. As melodias das músicas do compositor A são mais simples, as dinâmicas mais constantes, e as alterações ocorrem de forma mais gradual do que nas músicas do compositor B. Apesar das músicas de ambos os compositores apresentarem, em geral, características que permitem considerá-las como músicas relaxantes, as músicas do compositor A possuem maior quantidade de elementos composicionais relaxantes do que as músicas do compositor B.

\section{2) Análise de dados obtidos dos participantes}

As análises iniciais para observação da natureza e forma da distribuição dos dados, a partir da inspeção dos histogramas e dos dados de curtose e assimetria das variáveis relacionadas aos escores obtidos na HADS, PAPSI e PAPSII, permitiram verificar que, para a maioria dos casos, os dados são assimétricos e não seguem uma distribuição normal. Adicionalmente, o número de participantes de cada grupo é reduzido. Para as análises dos dados, como não foram satisfeitas as condições para os testes paramétricos, optou-se, portanto, pela utilização de testes estatísticos não paramétricos. Optou-se também por não ajustar os dados atípicos encontrados, uma vez que não exercem influência sobre a ordenação dos dados e sobre a mediana. Os dados obtidos a partir do cálculo de medidas de tendência central (média e mediana) e medidas de dispersão (desvio padrão) para os dados obtidos na HADS e cada componente do PAPSI e PAPSII separados por grupos ( $\mathrm{N}=18$, para cada grupo) são apresentados na Tabela 3.

A partir dos dados obtidos, considerando que resultados maiores ou iguais a 9 na escala de ansiedade da HADS apontam para sintomas de ansiedade, pode-se observar que todos os grupos apresentaram um alto índice nesta escala, sendo maior para o grupo que ouviu as músicas do compositor A. Identificou-se que a média de Raiva/Medo e Tristeza do PAPSII é menor do que a do PAPSI e a média de serenidade é maior no PAPSII do que no PAPSI.

Os dados da HADS foram correlacionados com os dados obtidos no PAPSI e PAPSII, utilizando-se o coeficiente de correlação de Spearman para verificar se o histórico de ansiedade se correlacionaria com os quatro componentes do PAPS 9 (Tabela 4).

Com base nos dados da Tabela 4, percebe-se que o fator ansiedade da HADS correlacionou-se positivamente com Raiva/Medo e Tristeza nas condições PAPSI e PAPSII e negativamente com Serenidade e Alegria. Por outro lado, o fator depressão da HADS correlacionou-se positivamente com os fatores de Raiva/Medo e Tristeza e negativamente com os fatores Alegria e Serenidade na condição PAPSI, enquanto que na condição PAPSII, somente apresentou uma correlação negativa com o fator Alegria.

Para verificar se os resultados obtidos pelos participantes no PAPSII, para cada condição (compositor A, compositor B, coletânea e controle), foram significativamente diferentes do PAPSI, para cada grupo, utilizou-se o teste estatístico Wilcoxon para medidas repetidas (Tabela 5).

A partir dos dados obtidos, pode-se observar que, para todos os grupos, os escores obtidos no PAPSII no fator Raiva/Medo, foram significativamente menores $(\mathrm{p}<0,05)$ do que os escores obtidos no PAPSI para todos os grupos. Os escores obtidos no PAPSII no fator Tristeza também apresentaram-se significativamente menores $(\mathrm{p}<0,05)$ do que os escores obtidos no PAPSI para todos os grupos, com exceção da condição Compositor B. Observa-se também que os escores obtidos no PAPSII no fator Serenidade foram significativamente maiores $(p<0,05)$ do que os escores obtidos no PAPSI para todos os grupos.

$O$ teste não paramétrico para amostras independentes de Kruskal-Wallis foi realizado para verificar se houve diferenças significativas entre as quatro 
diferentes condições (Compositor A, Compositor B, Coletânea e Controle) nas pontuações obtidas nas categorias do PAPSII. Como, em geral, houve diferenças entre as pontuações nas condições pré e pós-teste do PAPS, objetivou-se averiguar se as condições com audição de música diferiam da condição controle em algum aspecto específico do PAPSII. A Tabela 6 apresenta os resultados.

Tabela 3

Escores obtidos pelos participantes na HADS e PAPS.

\begin{tabular}{lccccccccccccc}
\hline & \multicolumn{3}{c}{ Compositor A } & \multicolumn{3}{c}{ Compositor B } & \multicolumn{3}{c}{ Coletânea } & \multicolumn{3}{c}{ Controle } \\
\cline { 2 - 11 } & Média & Med. & DP* & Média & Med. & DP* & Média & Med. & DP* & Média & Med. & DP* $^{*}$ \\
\hline $\begin{array}{l}\text { HADS } \\
\text { Ansiedade }\end{array}$ & 10.44 & 11.00 & 4.87 & 8.56 & 9.00 & 4.16 & 8.94 & 9.50 & 3.81 & 9.28 & 10.00 & 4.64 \\
$\begin{array}{l}\text { HADS } \\
\text { Depressão }\end{array}$ & 8.56 & 9.00 & 5.09 & 7.00 & 6.00 & 4.67 & 7.33 & 7.00 & 3.34 & 7.39 & 8.00 & 2.62 \\
$\begin{array}{l}\text { PAPSI } \\
\text { Raiva/Medo }\end{array}$ & 4.28 & 4.00 & 3.58 & 2.78 & 2.50 & 2.34 & 3.72 & 3.50 & 2.72 & 3.56 & 2.50 & 3.50 \\
$\begin{array}{l}\text { PAPSI } \\
\text { Alegria }\end{array}$ & 4.33 & 4.00 & 3.76 & 5.94 & 5.00 & 4.73 & 3.94 & 3.00 & 3.80 & 4.50 & 3.50 & 4.15 \\
$\begin{array}{l}\text { PAPSI } \\
\text { Tristeza }\end{array}$ & 3.28 & 2.50 & 3.56 & 1.83 & 0.50 & 2.53 & 1.06 & 1.00 & 1.55 & 2.44 & 1.50 & 3.13 \\
$\begin{array}{l}\text { PAPSI } \\
\text { Serenidade }\end{array}$ & 4.33 & 3.00 & 4.47 & 7.06 & 7.00 & 5.23 & 4.00 & 3.00 & 4.01 & 4.44 & 2.50 & 4.54 \\
$\begin{array}{l}\text { PAPSII } \\
\text { Raiva/Medo }\end{array}$ & 0.56 & 0.00 & 1.25 & 0.50 & 0.00 & 1.20 & 0.28 & 0.00 & 0.57 & 1.78 & 1.00 & 2.02 \\
$\begin{array}{l}\text { PAPSII } \\
\text { Alegria }\end{array}$ & 3.94 & 3.00 & 3.75 & 5.78 & 4.00 & 4.76 & 2.83 & 2.50 & 2.90 & 3.28 & 2.50 & 3.43 \\
$\begin{array}{l}\text { PAPSII } \\
\text { Tristeza }\end{array}$ & 0.94 & 0.00 & 2.39 & 0.94 & 0.00 & 2.36 & 0.17 & 0.00 & 0.38 & 1.11 & 0.00 & 2.14 \\
$\begin{array}{l}\text { PAPSII } \\
\text { Serenidade }\end{array}$ & 8.00 & 8.00 & 3.97 & 9.83 & 9.50 & 3.78 & 7.61 & 7.50 & 2.73 & 6.56 & 4.50 & 4.72 \\
\hline
\end{tabular}

PAPS: Protocolo de avaliação de percepções subjetivas; HADS: Escala Hospitalar de Ansiedade e Depressão; Med: Mediana; DP: Desvio padrão.

Tabela 4

Coeficientes de correlação ( $\rho$ de Spearman) e valores p para HADS e PAPS para o total da amostra $(\mathrm{N}=72)$.

\begin{tabular}{lcccc}
\hline & \multicolumn{2}{c}{ HADS Ansiedade } & \multicolumn{2}{c}{ HADS Depressão } \\
\cline { 2 - 5 } & $\rho$ & $\mathrm{p}$ & $\rho$ & $\mathrm{p}$ \\
\hline PAPS I Raiva/Medo & $0,474^{* *}$ & 0.001 & $0,288^{*}$ & 0.014 \\
PAPSzI Alegria & $-0,452^{* *}$ & 0.001 & $-0,507^{* *}$ & 0.001 \\
PAPSI Tristeza & $0,427^{* *}$ & 0.001 & $0,490^{* *}$ & 0.001 \\
PAPSI Serenidade & $-0,379^{* *}$ & 0.001 & $-0,412^{* *}$ & 0.001 \\
PAPSII Raiva/Medo & $0,235^{*}$ & 0.047 & 0.091 & 0.445 \\
PAPSII Alegria & $-0,333^{* *}$ & 0.004 & $-0,357^{* *}$ & 0.002 \\
PAPSII Tristeza & $0,302^{* *}$ & 0,010 & 0.161 & 0.176 \\
PAPSI Serenidade & $-0,308^{* *}$ & 0.008 & -0.191 & 0.109 \\
\hline
\end{tabular}

HADS: Escala Hospitalar de Ansiedade e Depressão; PAPS: Protocolo de avaliação de percepções subjetivas.

${ }^{* *} \mathrm{p}<0,01 ;{ }^{*} \mathrm{p}<0,05$ (bi-caudal). 
A partir dos dados obtidos, observa-se que houve diferença entre os grupos somente para a categoria Raiva/ Medo do PAPSII.Partindo-se deste resultado, averiguou-se se havia diferença entre as condições com audição de música somente para esta variável (Raiva/Medo PAPSII). Os resultados são apresentados na Tabela 7 .

Observa-se que não houve diferenças significativas entre os grupos experimentais em que os participantes foram submetidos à audição musical. Para especificar o resultado obtido a partir do Kruskal-Wallis, utili- zou-se o teste de independência não paramétrico U de Mann-Whitney para verificar se houve diferença significativa entre as medianas das pontuações obtidas pelos participantes na categoria Raiva/Medo do PAPSII entre cada grupo experimental e o grupo controle (Tabela 8).

Pelos resultados obtidos, pode-se observar que todos os grupos experimentais apresentaram diferenças significativas em relação ao grupo controle nos escores obtidos na PAPSII Raiva/Medo, com maior poder estatístico para a condição "Coletânea".

Tabela 5

Teste de Wilcoxon para verificar a diferença entre os resultados obtidos na PAPSI e PAPSII.

\begin{tabular}{|c|c|c|c|c|c|c|c|c|c|c|c|c|c|}
\hline & \multicolumn{5}{|c|}{ Raiva/ Medo } & \multicolumn{3}{|l|}{ Alegria } & \multicolumn{2}{|l|}{ Tristeza } & \multicolumn{3}{|c|}{ Serenidade/ Relaxamento } \\
\hline & $\begin{array}{c}\mathrm{PN} / \mathrm{PP} \\
\text { (a) }\end{array}$ & $\begin{array}{l}\text { Posto } \\
\text { médio }\end{array}$ & Escore $\mathrm{z}$ & Valor $\mathrm{p}$ & $\begin{array}{l}\text { Posto } \\
\text { médio }\end{array}$ & Escore z & $\begin{array}{c}\text { Valor } \\
\mathrm{p}\end{array}$ & $\begin{array}{l}\text { Posto } \\
\text { médio }\end{array}$ & Escore z & Valor $\mathrm{p}$ & $\begin{array}{l}\text { Posto } \\
\text { médio }\end{array}$ & Escore z & Valor $\mathrm{p}$ \\
\hline \multirow{2}{*}{$\begin{array}{l}\text { Compositor } \\
\text { A }\end{array}$} & PN & 9 & - & - & 9.22 & - & - & 6.68 & - & - & 4.00 & $-3,319(c)$ & $0,001^{*}$ \\
\hline & $\mathrm{PP}$ & 0 & $-3,633(b)$ & $0,001^{*}$ & 7,57 & $-0,785(b)$ & 0,432 & 4,50 & $-2,714(b)$ & $0,001^{*}$ & 8,80 & - & - \\
\hline \multirow{2}{*}{$\begin{array}{l}\text { Compositor } \\
\text { B }\end{array}$} & $\mathrm{PN}$ & 8,69 & - & - & 6,71 & - & - & 5,86 & - & - & 2,75 & $-2,959(\mathrm{c})$ & $0,003^{*}$ \\
\hline & $\mathrm{PP}$ & 3.5 & $-3,035(b)$ & $0,007^{*}$ & 7,33 & $-0,105(b)$ & 0,916 & 4,67 & $-1,381(b)$ & 0,167 & 8,29 & - & - \\
\hline \multirow{2}{*}{ Coletânea } & PN & 8,50 & - & - & 8,15 & - & - & 6,33 & - & - & 0 & $-3,425(c)$ & $0,001^{*}$ \\
\hline & $\mathrm{PP}$ & 0 & $-3,530(b)$ & $0,001^{*}$ & 5,88 & $-1,835(b)$ & 0,066 & 4,50 & $-2,230(b)$ & $0,026^{* *}$ & 8,00 & - & - \\
\hline \multirow{2}{*}{ Controle } & PN & 7,70 & - & - & 6,90 & - & - & 7,00 & - & - & 5,67 & $-2,456(\mathrm{c})$ & $0,014^{* *}$ \\
\hline & $\mathrm{PP}$ & 4.67 & $-2,218(b)$ & $0,027^{* *}$ & 7,33 & $-1,673(b)$ & 0,094 & 4,00 & $-2,490(b)$ & $0,013^{* *}$ & 8,58 & - & - \\
\hline
\end{tabular}

(a)PN=Posto negativo; PP=Posto positivo; (b) Baseado em postos positivos; (c) Baseado em Postos negativos; ${ }^{*} \mathrm{p}<0,01$; ${ }^{* *} \mathrm{p}<0,05$.

Tabela 6

Teste Kruskal-Wallis para verificar diferenças entre as condições nos escores obtidos no PAPSII.

\begin{tabular}{lccc}
\hline Variáveis & $\chi^{2}$ & Df & Valor $\mathrm{p}$ \\
\hline HADS Ansiedade & 1.641 & 3 & 0,650 \\
HADS Depressão & 2.798 & 3 & 0.424 \\
PAPSI Raiva/ Medo & 1.946 & 3 & 0.584 \\
PAPSI Alegria & 1.863 & 3 & 0.601 \\
PAPSI Tristeza & 4.007 & 3 & 0.261 \\
PAPSI Serenidade/ Relaxamento & 4.238 & 3 & 0.237 \\
PAPSII Raiva/ Medo & 10.235 & 3 & $0,017^{*}$ \\
PAPSII Alegria & 5.109 & 3 & 0.164 \\
PAPSII Tristeza & 2.306 & 3 & 0.511 \\
PAPSII Serenidade/ Relaxamento & 6.158 & 3 & 0.104 \\
\hline
\end{tabular}

${ }^{*} \mathrm{p}<0,05$ 
Tabela 7

Teste Kruskal-Wallis para verificar se houve diferenças entre os grupos experimentais nos escores obtidos no PAPSII Raiva/Medo.

\begin{tabular}{lccccc}
\hline Variável & Condição & Posto Médio & $\chi 2$ & df & Valor p \\
\hline \multirow{3}{*}{ PAPSII Raiva/ Medo } & Compositor A & 28.22 & 0.236 & 2 & 0.889 \\
\cline { 2 - 6 } & Compositor B & 27.89 & - & - & - \\
\cline { 2 - 6 } & Coletânea & 26.39 & - & - & - \\
\hline
\end{tabular}

Tabela 8

Teste U de Mann-Whitney para verificar se houve diferenças entre as medianas de cada grupo experimental e o grupo controle para a categoria Raiva-medo do PAPSII.

\begin{tabular}{lcccccc}
\hline Condições & Posto Médio & Soma dos postos & Ude Mann-Whitney & Wilcoxon W & Escore Z & Valor p \\
\hline Compositor A & 14.97 & 269.5 & 98.5 & 269.5 & -2.215 & $0,027^{* *}$ \\
Controle & 22.03 & 396.5 & - & - & - & - \\
Compositor B & 14.81 & 266.5 & 95.5 & 266.5 & -2.323 & $0,020^{* *}$ \\
Controle & 22.19 & 399.5 & - & - & - & - \\
Coletânea & 14.28 & 257.0 & 86.0 & 257.0 & -2.695 & $0,007^{*}$ \\
Controle & 22.72 & 409.0 & - & - & - & - \\
\hline
\end{tabular}

${ }^{*} \mathrm{p}<0,01 ;{ }^{* *} \mathrm{p}<0,05$.

\section{Discussão}

A partir da análise qualitativa das músicas utilizadas para indução de relaxamento compostas pelo compositor A e pelo compositor B, verificou-se que todas as músicas apresentaram características de músicas consideradas relaxantes (Khalfa et al., 2003; Krout, 2007; Riccardi, 2002), tais como andamento lento, regularidade de tempo e harmonia, dinâmica leve com pouca variação, timbre suave, combinação harmoniosa de instrumentos, melodias com legatos (com notas ligadas, sem interrupção), harmonia e progressões de acordes mais simples. Porém, em geral, as músicas do Compositor A apresentaram maior número de características relaxantes do que as músicas do Compositor B. Além disso, nas músicas do Compositor B, a modulação frequente do modo maior para o modo menor, pode suscitar emoções mais associadas à tristeza do que à serenidade (Bigand et al., 2005), o que pode ter contribuído também para o resultado não significativo encontrado para a diferença entre o PAPSI e PAPSII no fator Tristeza, somente nesta condição.

Todos os grupos participantes do estudo apresentaram alto índice na escala de ansiedade da HADS, o que aponta para sintomas de ansiedade. Deve-se salientar que os participantes foram recrutados em uma população composta por clientes da TIP Clínica em busca de tratamento terapêutico pelo método ADI/TIP, o que possivelmente contribuiu para os altos índices apresentados. Considerando a relação entre os fatores da HADS e do PAPS, ponderando que o fator Ansiedade correlacionou-se positivamente com Raiva e Tristeza e negativamente com Serenidade e Alegria nas condições PAPSI e PAPSII, pode-se inferir que os escores referentes a sintomas de ansiedade apresentaram-se mais relacionados à valência (positiva ou negativa), do que à excitabilidade (alta ou baixa) nas condições apresentadas no PAPS (Bigand et al., 2005). Quanto ao fator depressão da HADS, este apresentou relação negativa tanto na condição pré-teste (PAPSI) quanto na condição pós-teste (PAPSII) somente para os itens relacionados à Alegria no PAPS. Na condição pré-teste, o fator Depressão da HADS também correlacionou-se positivamente com os fatores de Raiva/Medo e Tristeza e negativamente com o fator Serenidade, apresentando-se, portanto, mais relacionado à valência do que à excitabilidade em relação aos fatores considerados no PAPS.

Os resultados obtidos pelos participantes no PAPSII, para cada condição (Compositor A, Compositor B, Coletânea e Controle), foram significativamente maiores $(p<0,05)$ do que no PAPSI no fator Serenidade/Relaxamento, para todos os grupos e significativamente menores $(p<0,05)$ do que no 
PAPSI, para todos os grupos, no fator Raiva/Medo e também no fator Tristeza, mas neste último com exceção da condição Compositor B. Portanto, depois de submetidos às condições estabelecidas no estudo, observa-se que houve: 1) uma diminuição significativa de marcações para os itens relacionados à Raiva/Medo; 2) uma diminuição significativa de marcações para os itens relacionados à Tristeza (com exceção da condição Compositor B); 3) um aumento significativo no número de marcações referentes aos itens relacionados à Serenidade/ Relaxamento e 4) não houve diferença entre o PAPSI e PAPSII para os itens relacionados à Alegria. A partir disto, pode-se inferir que, independentemente da condição, os participantes apresentaram uma percepção subjetiva de diminuição dos sentimentos relacionados ao fator Raiva/Medo e aumento dos sentimentos relacionados ao fator Serenidade, independentemente do uso de música ou não. Apesar de ter havido diferenças entre as pontuações nas condições pré e pós-teste do PAPS para todas as condições, inclusive a condição controle, a partir do teste não paramétrico para amostras independentes de Kruskal-Wallise e do teste de independência não-paramétrico $U$ de Mann-Whitney, averiguou-se que as condições experimentais com audição de música diferiram da condição controle somente para a categoria Raiva/Medo do PAPSII, indicando que a diminuição da marcação para os itens relacionados à Raiva/Medo (excitabilidade alta e valência negativa) foi significativamente maior nos grupos experimentais em relação ao grupo controle. Os grupos experimentais não apresentaram diferenças entre si, mas o grupo referente à audição das músicas da "Coletânea" apresentou um maior poder estatístico. Isto indica que, apesar de não haver um aumento de emoções relacionadas ao fator Serenidade em relação à condição controle, a audição de músicas com característica relaxantes contribuiu para redução de emoções relacionadas ao fator Raiva/Medo, que é oposto ao fator Serenidade e corresponde a uma valência negativa e excitabilidade alta. Esta percepção por parte dos participantes de redução da excitabilidade emocional observada vai de encontro aos estudos que indicam que músicas com características relaxantes podem contribuir para a diminuição do estresse e promoção de estados de relaxamento (Nuki et al., 1999; Khalfa et al., 2003; Kreutz et al., 2004; Krout, 2007).
Portanto, pode-se dizer que, apesar das músicas do Compositor A apresentarem mais características relaxantes do que as do Compositor B, e das músicas deste Compositor B conterem elementos que podem suscitar emoções relacionadas à tristeza, todas as músicas analisadas podem ser consideradas estruturalmente como relaxantes, a partir de suas características composicionais, e tiveram efeito para redução da pontuação da categoria de Raiva/Medo no PAPS.

\section{Considerações finais}

Tendo em vista o objetivo deste estudo de avaliar os efeitos das músicas utilizadas para promoção de relaxamento e redução da ansiedade, na fase preparatória do Método ADI/TIP, pode-se dizer que as músicas utilizadas possuem as características específicas consideradas relaxantes, tais como andamento lento, regularidade de tempo e harmonia, dinâmica leve e com pouca variação, timbre suave, combinação harmoniosa de instrumentos, melodias com legatos, harmonia e progressões de acordes mais simples, e contribuem para induzir o paciente ao estado de relaxamento necessário ao processo terapêutico. A fase preparatória da ADI/TIP se constitui em uma etapa fundamental da terapia, pois, este método pressupõe que através da promoção do relaxamento e diminuição da ansiedade dos pacientes, pode-se acessar mais facilmente o inconsciente através do consciente. De acordo com o método, isto só se torna possível a partir da redução do estado de alerta e de abertura do paciente ao inusitado. Por isso, na sua fase preparatória, o Método da ADI/TIP utiliza-se da música para essa finalidade.

$\mathrm{Na}$ comparação das músicas usadas na fase preparatória, observou-se que as três músicas do compositor A são mais constantes, sem grandes modificações e com timbres considerados mais relaxantes do que as três músicas do compositor B. Ainda que nas músicas deste Compositor B se encontrem elementos que podem suscitar emoções relacionadas à tristeza, conclui-se que todas as músicas analisadas, tanto as do Compositor A quanto as do Compositor B podem ser consideradas estruturalmente como relaxantes. Uma vez que músicas consideradas relaxantes possuem características específicas, pondera-se que a escolha das músicas para as sessões de relaxamento deve considerar tais características, caso contrário o propósito não será alcançado. 
Conclui-se, portanto, que as músicas utilizadas como recurso auxiliar na fase preparatória do Método ADI/TIP, consideradas como relaxantes, contribuem para diminuição de sensações correspondentes a uma valência negativa e excitabilidade alta, favorecendo a promoção do estado de relaxamento necessário à efetividade desse método terapêutico. Estas músicas podem ter a propriedade de quebrar o estresse contínuo e diminuir a ansiedade, o que consequentemente favorece a liberação de endorfinas e a redução do cortisol. Estudos futuros que utilizem uma medida de cortisol podem inves-

\section{Referências}

Andrade, L. H. S. G., \& Gorestein, C. (1998). Aspectos gerais das escalas de avaliação de ansiedade. Revista de Psiquiatria Clínica, 25, 285-290. Recuperado de http://www.hcnet.usp.br/ipq/revista/vol26/vol25/n6/ansi256a.htm

Andrade, M. C., \& Ávila, A. O. V. (2007). O uso da música na prática da atividade física. Revista Tecnicouro, 72-75. Recuperado de http://www.tecnicouro.com. br/227/materias/227_ac.pdf

Bigand, E., Vieillard, S., Madurell, F., Marozeau, J., \& Dacquet, A. (2005). Multidimensional scaling of emotional responses to music: the effect of musical expertise and of the duration of the excerpts. Cognition \& Emotion , 19(8), 1113-1139. doi:10.1080/02699930500204250

Botega, N. J., Bio, M. R., Zomignani, M. A., Garcia Junior, C., \& Pereira, W. A. B. (1995). Transtornos do humor em enfermaria de clínica médica e validação de escala de medida (HAD) de ansiedade e depressão. Revista Saúde Pública, 29(5), 355-63. doi:10.1590/S0034-89101995000500004

Botega, N. J., Pondé, M. P., Medeiros, P., Lima, M. G., \& Guerreiro, C. A. M. (1998). Validação da escala hospitalar de ansiedade e depressão (HAD) em pacientes epiléticos ambulatoriais. Jornal Brasileiro de Psiquiatria, 47(6), 285-289.

Chanda, M. L., \& Levitin, D. J. (2013). The neurochemistry of music. Trends in Cognitive Sciences, 17(4), 179-193. doi:10.1016/j.tics.2013.02.007

Chafin S., Roy, M., Gerin, W., \& Christenfeld, N. (2004). Music can facilitate blood pressure recovery from stress. British Journal of Health Psychology, 9(3), 393-403. doi:10.1348/1359107041557020 tigar se estas alterações fisiológicas realmente ocorrem para estas músicas.

Por fim, pode-se observar que, na fase preparatória para a ADI/TIP, a ausência da música, não inviabiliza o prosseguimento do processo. Porém, a audição de músicas com características específicas, nessa fase, potencializa os efeitos de relaxamento e das sugestões de imagens positivas que são utilizadas concomitantemente à audição musical. Portanto, a utilização das músicas compostas por ambos os compositores favorece a indução do relaxamento e são adequadas e recomendáveis para essa fase de aplicação do Método ADI/TIP.

Chuang, C. Y., Han, W. R., Li, P. C., \&Young, S. T. (2010). Effects of music therapy on subjective sensations and heart rate variability in treated cancer survivors: a pilot study. Complementary Therapies in Medicine, 18(5), 224-226. doi:10.1016/j.ctim.2010.08.003

Ferreira, C. C. M., Remedi, P. P., \& Lima, R. A. G. (2006). A música como recurso no cuidado à criança hospitalizada: uma intervenção possível? $R e$ vista Brasileira de Enfermagem, 59(5): 689-693. doi:10.1590/S0034-71672006000500018

Good, M. (1995). A comparison of the effects of jaw relaxation and music on postoperative pain. Nursing Research, 44(1): 52-57. Recuperado de http:// journals.lww.com/nursingresearchonline/Abstract/1995/01000/A_Comparison_of_the_Effects_Of_ Jaw_Relaxation_and.10.aspx

Gruzzelier, J. H. (2002). A review of the impact of hypnosis, relaxation, guided imagery and individual differences on aspects of immunity and health. Stress, 5(2): 147-163. doi:10.1080/10253890290027877

Hatem, T. P., Lira P. I. C., \& Mattos, S.S. (2006). Efeito terapêutico da música em crianças em pós-operatório de cirurgia cardíaca. Jornal de Pediatra, 82(3): 186-192. doi:10.2223/JPED.1473

Horne-Thompson, A., \& Grocke, D. (2008). The effect of music therapy on anxiety in patients who are terminally ill. Journal of Palliative Medicine, 11(4), 582-590. doi:10.1089/jpm.2007.0193

Kain Z. N., Wang, S. M., Mayes, L.C., Krivutza, D. M., \& Teague, B. A. (2001). Sensory stimuli and anxiety in children undergoing surgery: a randomized, controlled trial. Anesthesia \& Analgesia, 92(4), 897-903 
Klainin-Yobas, P., Oo, W. N., Suzanne Yew, P. Y., \& Lau, Y. (2015). Effects of relaxation interventions on depression and anxiety among older adults: a systematic review. Aging \& Mental Health, 19(12): 1043-1055. doi:10.1080/13607863.2014.997191

Khalfa, S., Dalla-Bella, S., Roy, M., Peretz, I., \& Lupien, S. J. (2003). Effects of relaxing music on salivary cortisol level after psychological stress. Annals of the New York Academy of Science, 999, 374-376. doi:10.1196/annals.1284.045

Koelsch, S., Fuermetz, J., Sack, U., Bauer, K., Hohenadel, M., Wiegel, M., ... Heinke, W. (2011). Effects of music listening on cortisol levels and propofol consumption during spinal anesthesia. Frontiers in Psychology, 2, 58. doi:10.3389/fpsyg.2011.00058

Kreutz, G., Bongard, S., Rohrmann, S., Hodapper, V., \& Grebe, D. (2004). Effects of choir singing or listening on secretory immunoglobulin A, cortisol, and emotional state. Journal of Behavioral Medicine, 27(6), 623-635. doi:10.1007/s10865-004-0006-9

Krout, R.E. (2007). Music listening to facilitate and promote wellness: Integrated aspects of four neurophysiological responses to music. The Arts in Psychotherapy, 34(2): 134-141. doi:10.1016/j.aip.2006.11.001

Madson, A. T., \& Silverman, M. J. (2010). The effect of music therapy on relaxation, anxiety, pain perception, and nausea in adult solid organ transplant patients. Journal of Music Therapy, 47(3), 220-232. doi:10.1093/jmt/47.3.220

Marcolino, J. A. M., Mathias, L. A. S. T, Piccinini Filho, L., Guaratini, A. A., Suziki, F. M., \& Alli, L. A. C. (2007). Escala hospitalar de ansiedade e depressão: estudo da validade de critério e da confiabilidade com pacientes no pré-operatório. Revista Brasileira de Anestesiologia, 57(1), 52-62. doi:10.1590/S0034-70942007000100006

Michopoulos, I., Douzenis, A., Kalkavoura, C., Christodoulou, C., Michalopoulou, P., Kalemi, G., ... Lykouras, L. (2008). Hospital Anxiety and Depression Scale (HADS): validation in a Greek general hospital sample. Annals of General Psychiatry, 7, 4. doi:10.1186/1744-859X-7-4

Moraes, R. J. (2009). As chaves do inconsciente (25a ed.). Petrópolis, RJ: Vozes.

Moraes, R. J. (2007). O inconsciente sem fronteiras (11a ed.). Aparecida, SP: Santuário.

Najafi Ghezeljeh, T., Mohades Ardebili, F., Rafii, F., \& Haghani. H. (2016). The effects of music inter- vention on background pain and anxiety in burn patients: randomized controlled clinical trial. Journal of Burn Care \& Research, 37(4): 226-234. doi:10.1097/BCR.0000000000000266

Nuki, M., Yoshiuchi, K. M. D., \& Nomura, S. M. (1999). Effects of healing music on endocrinological changes. In Proceedings of the 9th World Congress for $\mathrm{Mu}$ sic Therapy (pp. 124-135). Washington, DC.

Peterson, E. M., \& Silva, L. B. (2006). Musicoterapia em oncologia: uma experiência de humanização no hospital do câncer HC II - Inca - Rio de Janeiro. In: Proceedings of the 120 Simpósio Brasileiro de Musicoterapia, 6o Encontro Nacional de Pesquisa em Musicoterapia, 20 Encontro Nacional de Docência em Musicoterapia. Goiânia, GO. Recuperado de http://www.sgmt.com.br/anais12trabalhos.html

Primo, C. C., \& Amorim, M. H. C. (2008). Efeitos do relaxamento na ansiedade e nos níveis de IgA salivar de puérperas. Revista Latino-Americana de Enfermagem, 16(1), 36-41. doi:10.1590/S0104-11692008000100006

Riccardi, P. L. S. (2002). Musicoterapia y parálisis cerebral. In: Confederación ASPACE, F.E.I.S.D, Confederación Autismo España, editors. Programa de formación para mediadores em musicoterapia y discapacidad: libro de ponencias (pp. 77-128). Madrid: The editors. Recuperado de http://sid.usal.es/libros/discapacidad/6823/8-4-2/programa-de-formacion-para-mediadores-en-musicoterapia-y-discapacidad-musicoterapia-2002.aspx

Russel, J. (1980). A circumplex model of affect. Journal of Personality and Social Psychology, 39(6), 1161-1178.

Snaith, R. P. (2003). The Hospital Anxiety And Depression Scale. Health and Quality of Life Outcomes, 1, 29. doi:10.1186/1477-7525-1-29

Souza, L. P. M., Forgione, M. C. R., \& Alves, V. L. R. (2000). Técnicas de relaxamento no contexto da psicoterapia de pacientes com queixas de dor crônica e fibromialgia: uma proposta. Acta Fisiátrica, 7(2), 56-60. Recuperado de http:// www.actafisiatrica.org. br/detalhe_artigo.asp?id=323

Taets, G. G. D. C., Borba-Pinheiro, C. J., Figueiredo, N. M. A. D., \& Dantas, E. H. M. (2013). Impact of a music therapy program on the stress level of health professionals. Revista Brasileira de Enfermagem, 66(3), 385-390. doi:10.1590/S0034-71672013000300013 
Tan, X., Yowler, C. J., Super, D. M., \& Fratianne, R. B. (2010). The efficacy of music therapy protocols for decreasing pain, anxiety, and muscle tension levels during burn dressing changes: a prospective randomized crossover trial. Journal of Burn Care \& Research, 31(4), 590-597. doi:10.1097/BCR.0b013e3181e4d71b

Thoma, M. V., La Marca, R., Brönnimann, R., Finkel, L., Ehlert, U., \& Nater, U. M. (2013). The effect of music on the human stress response. PloS One, 8(8), e70156. doi:10.1371/journal.pone.0070156

Vera, M. N., Vila, J. (2002). Técnicas de relaxamento. In: Caballo, V. E. Manual de técnicas de terapia e modificação do comportamento (2a ed.). (pp.147-165). São Paulo: Santos.

Wang, S.M., Kulkarni, L., Dolev, J, \& Kain, N. Z. (2002). Music and preoperative anxiety: a randomized, controlled study. Anesthesia \& Analgesia, 94(6), 14891494. doi:10.1213/00000539-200206000-00021

Marília Nunes Silva

Doutora. Docente da Universidade do Estado de Minas Gerais, Belo Horizonte - BH. Brasil.

E-mail: marilianunespsi@gmail.com

Ana Carolina Duarte Valadares

Fundação de Saúde Integral Humanística FUNDASINUM

E-mail: carolina.duarte@fundasinum.org.br

Gerlaine Teixeira Rosa

Fundação de Saúde Integral Humanística FUNDASINUM

E-mail: rosa.gerlaine@gmail.com

\section{Liliane Cristina Moreira Lopes}

Fundação de Saúde Integral Humanística FUNDASINUM

E-mail: liliane.moreira@sga.pucminas.br

Célia Auxiliadora dos Santos Marra

Fundação de Saúde Integral Humanística FUNDASINUM

E-mail: ceadipsi@gmail.com.br

Endereço para envio de correspondência:

Fundação de Saúde Integral Humanística (FUN-

DASINUM), Departamento de Pesquisa. Rua Musas,

n. 166, Santa Lucia. CEP.: 30360-660. Belo

Horizonte - MG. Brasil.

Recebido 18/12/2014

Reformulação 08/06/2015

Aprovado 19/07/2016

Received 12/18/2014

Reformulated 06/08/2015

Approved 07/19/2016

Recibido 18/12/2014

Reformulado 08/06/2015

Aceptado 19/07/2016

Como citar: Nunes-Silva, M., Valadares, A. C. D., Rosa, G. T., Lopes, L C. M., \& Marra, C. A S. (2016). Avaliação de músicas compostas para indução de relaxamento e de seus efeitos psicológicos. Psicologia: Ciência e Profissão, 36(3): 709-725. doi:10.1590/1982-3703001672014

How to cite: Nunes-Silva, M., Valadares, A. C. D., Rosa, G. T., Lopes, L C. M., \& Marra, C. A S. (2016). Evaluation of music composed for inducing relaxation and its psychological effects. Psicologia: Ciência e Profissão, 36(3): 709-725. doi:10.1590/1982-3703001012014

Cómo citar: Nunes-Silva, M., Valadares, A. C. D., Rosa, G. T., Lopes, L C. M., \& Marra, C. A S. (2016). Evaluación de canciones compuestas para la inducción a la relajación y sus efectos psicológicos. Psicologia: Ciência e Profissão, 36(3): 709-725. doi:10.1590/1982-3703001012014 